\title{
Multiscale unveil of moisture in buildings
}

\author{
Jan Carmeliet*,a \\ aChair of Building Physics, Department of Mechanical and Process Engineering, Zurich, Switzerland
}

\begin{abstract}
In this lecture, we zoom at different scales unveiling different aspects of moisture in buildings. At atomistic scale, we unravel sorption hysteresis in wood. At pore scale, we analyze the impact of particle deposition on the drying process of a porous material. At material scale, we present a two-phase model for predicting the effective moisture transport properties of books. At building scale, we analyze the potential of desorption cooling.

Peer-review under the responsibility of the organizing committee of the ICMB21.
\end{abstract}

Keywords: Multscale modelling, sorption hysteresis, drying, moisture buffering, sorption cooling

\section{Introduction/Background}

In this presentation, we first zoom on the water sorption process at microscale using molecular dynamics, with a focus on understanding of sorption hysteresis [1], [2]. As an example, we discuss the impact of sorption hysteresis of finishing materials on the fluctuations in relative humidity inside a room.

A second zoom is on the drying of porous materials at pore scale using Lattice Boltzmann modelling (LBM) and a hybrid method using pore network modelling and LBM [6]. As an example, we discuss the drying of pure water and water with suspended particles from a porous medium. Drying of water shows mainly a first drying regime, while the drying of the suspension shows a second drying phase with decrease in drying rate. We analyze the deposition pattern of the particles and possible clogging of the pore system [5].

A third zoom is on material scale, where we analyze the moisture buffering effect of papers, books and textiles [3], [4]. Book is considered as a periodic system of parallel paper sheets and air layers. Effective moisture transport properties are determined considering two diffusion paths, one normal to the paper edge and one via the air layer normal to the paper sheet. The impact on average and maximum relative humidity of books and textiles is presented.

A final zoom is on building scale. We analyze the potential of precooling and of water desorption from inside hygroscopic materials as passive cooling measure for indoor sleeping rooms during heat waves [7].

\section{References}

[1] Mingyang Chen, Benoit Coasne, Robert Guyer, Dominique Derome and Jan Carmeliet, Role of hydrogen bonding in hysteresis observed in sorptioninduced swelling of soft nanoporous polymers, Nature Communications, vol. 9, pp. 3507, London: Nature Publishing Group, 2018.

[2] Mingyang Chen, Benoit Coasne, Robert Guyer, Dominique Derome and Jan Carmeliet, Molecular Simulation of Sorption-Induced Deformation in Atomistic Nanoporous Materials, Langmuir, vol. 35: no. 24, pp. 7751-7758, Washington, DC: American Chemical Society, 2019

[3] Hannelore Derluyn, Role of textiles and paper for stabilizing the indoor environment, master thesis KULeuven, 2006.

[4] H Derluyn, H Janssen, J Diepens, D Derome, J Carmeliet, Hygroscopic behavior of paper and books, Journal of Building Physics 31 (1), 9-34, 2007.

[5] Feifei Qin, Ali Mazloomi Moqaddam, Qinjun Kang, Dominique Derome and Jan Carmeliet, LBM Simulation of Self-Assembly of Clogging Structures by Evaporation of Colloidal Suspension in 2D Porous Media, Transport in Porous Media, vol. 128: no. 3, pp. 929-943, Berlin: Springer, 2019

[6] Jianlin Zhao, Feifei Qin, Qinjun Kang, Dominique Derome, Jan Carmeliet, Pore-scale Simulation of Drying in Porous Media Coupling Lattice Boltzmann Method and Pore Network Model, ready for submission, 2020

[7] Xiaohai Zhou, Jan Carmeliet, Matthias Sulzer and Dominique Derome, Energy-efficient mitigation measures for improving indoor thermal comfort during heat waves. Applied Energy, vol. 278, pp. 115620, New York, NY: Elsevier, 2020

\footnotetext{
* Corresponding author. cajan@ethz.ch
} 